\title{
MfERG responses to long-duration white stimuli in glaucoma patients
}

\author{
Margarita G. Todorova • \\ Anja M. Palmowski-Wolfe
}

Received: 25 August 2009/ Accepted: 9 February 2011/Published online: 22 February 2011

(C) Springer-Verlag 2011

\begin{abstract}
The intent of our study was to evaluate whether the response to a long-duration white stimulus in the multifocal electroretinogram (mfERG) is sufficiently sensitive to detect early retinal dysfunction in glaucoma. On-off mfERGs were recorded from 15 NTG and 15 HTG patients and compared with 14 control subjects. Recording parameters were the following: LED stimulus screen (RETIscan ${ }^{\mathrm{TM}}$ ), 100-ms stimulus duration, 200-ms stimulus interval, 11-min total recording time, stimulus matrix of 61 elements, frame rate: $70 \mathrm{~Hz}, \mathrm{Lmax}: 180 \mathrm{~cd} / \mathrm{m}^{2}$, Lmin: $0 \mathrm{~cd} / \mathrm{m}^{2}$, and filter setting: 1-200 Hz. The second negative response following stimulus onset (N2-on), as well as following stimulus offset (N2-off), was analyzed as an overall response and in quadrants, as well as in 4 small central and four adjoining peripheral areas per quadrant. The latency of the N2-on was significantly delayed in HTG in all response averages tested, while in NTG this was only seen in the overall response and in the small central response averages $(P<0.05)$. The most sensitive measure in HTG was the latency of the $\mathrm{N} 2$-on of the small peripheral response average of the superior temporal quadrant with an area under the ROC curve of 0.881 . For NTG, the most representative measure was the latency of the N2-on of the small central response average of the inferior nasal quadrant
\end{abstract}

M. G. Todorova $(\bowtie)$ · A. M. Palmowski-Wolfe Department of Ophthalmology, University Hospital Basel, Mittlere Strasse 91, 4031 Basel, Switzerland e-mail: todorovam@uhbs.ch with an area under the ROC curve of 0.793 . Our results showed that in stimulation with long-duration flashes, the second negative response following the on response, representative of the early $\mathrm{PhNR}$, is affected in glaucoma where N2-on showed a latency delay in POAG patients. The latency delay of the N2-on was more prominent for HTG than for NTG.

Keywords Light-emitting diode screen .

On-off mfERG responses .

Long-duration white flashes · Glaucoma

$\begin{array}{ll}\text { Abbreviations } & \\ \text { HTG } & \text { High-tension glaucoma } \\ \text { LED } & \text { Light-emitting diode screen } \\ \text { MD } & \text { Mean defect } \\ \text { NTG } & \text { Normal-tension glaucoma } \\ \text { N2-on } & \text { N2-on response } \\ \text { N2-off } & \text { N2-off response } \\ \text { Off-response } & \text { Response, following stimulus offset } \\ \text { On-response } & \text { Response, following stimulus onset } \\ \text { POAG } & \text { Primary open-angle glaucoma } \\ \text { PhNR } & \text { Photopic negative response } \\ \text { ROC } & \text { Receiver operating characteristic }\end{array}$

\section{Introduction}

Primary open-angle glaucoma (POAG) is a progressive optic neuropathy. In early stages, the proximal, i.e., the inner retinal layers, namely the ganglion cells 
are affected [1]. A number of clinical studies have attempted to clarify which components of the ERG reflect mainly inner retinal activity and also under which recording and processing conditions these can be enhanced.

In the Ganzfeld ERG, the photopic negative response $(\mathrm{PhNR})$ is a cornea-negative cone-driven deflection following the "b-wave" of the Ganzfeld photopic ERG, which has recently been studied as a possible new and sensitive measure to detect early glaucomatous dysfunction [2-5]. In the uniform ERG to luminance increment followed by a decrement, the PhNR is supposed to be of similar origin as the N95response of the pattern ERG (PERG), which is mainly generated by ganglion cells and their axons [6]. This is supported by pharmacological blockage studies: following intravitreal injection of TTX, the PhNR disappeared when brief or long-duration white stimuli on a white background were used [2]. In agreement with an inner retinal origin, the PhNR to stimuli of various durations is also markedly diminished in experimental glaucoma. Indeed, the PhNR-amplitudes were found to be greatly diminished, even when the visual field loss was only mild to moderate [2].

As POAG is a disease affecting primarily the ganglion cells in the inner retina [7, 8], further attempts have been made to assess whether the PhNR holds promise for the clinical evaluation of retinal function in patients with glaucoma. This seems to be the case as in glaucoma patients the PhNR to flashes of brief and of long-duration was markedly reduced as well, even in the presence of normal a- and b-waves $[3,4,9,10]$. In contrast, another study applying yet different stimulus conditions did not find significant changes in the PhNR of glaucoma patients [11].

As the full-field photopic ERG represents a summed response from the nerve fiber layer across the entire retina, localized changes, as for example in early glaucoma, could easily be missed. For example, a study, in primates with focal ganglion cell damage induced by a laser burn, compared a photopic fullfield ERG with a red stimulus on a rod-saturated blue background to mfERG recordings with a stimulus base interval of $106 \mathrm{~ms}$ [12]. Here, the amplitude of the PhNR of the full-field ERG did not show the focal damage. Therefore, a focal PhNR has been elicited to assess selectively localized areas of the retina $[4,6]$. Results of studies on focal ERGs in glaucomatous eyes showed attenuation of the PhNR, even when the disease affected the inner retinal function only locally $[4,5,13,14]$. This held true when responses were obtained from the macular, as well as from the paramacular retinal areas, for instance, from the superior temporal and inferior temporal areas, which are supposed to be most affected by glaucoma [14]. In the slow 7F sequence mfERG, in which standard waves of the Ganzfeld ERG can be seen [15], the local ganglion cell damage was also reflected in form of focally reduced PhNR-amplitudes and also slightly increased latencies in the area of damage.

Thus, the multifocal electroretinogram (mfERG) offers an objective test for evaluating and monitoring the local functional status of the retina with a high spatial resolution [16-19].

Changing the stimulus paradigms from a fast sequence $[20,21]$ to parameters that allow "standard" a- and b-waves to form and also increase inner retinal contributions to the mfERG [22-25] have greatly increased the sensitivity of the mfERG to detect glaucomatous damage.

Using a stimulus, "on" duration of $100 \mathrm{~ms}$ and an "off" duration of $100 \mathrm{~ms}$ ("on" and "off"responses), the photopic ERG [26, 27] and the photopic mfERG [28, 29] responses show an onresponse following the onset phase of the stimulus and an off-response following the offset of the stimulus. In the Ganzfeld photopic ERG, the on- and the offresponses are each followed by a negative trough, the PhNR-on and the PhNR-off, respectively. By averaging the PhNR-on and the PhNR-off of discrete long photopic flashes, the N95 of the PERG can be simulated, thus pointing to the same origin of these components [6]. This suggests that under photopic conditions, the negative troughs following the on- and off-responses may contain information that is also contained in the PhNR.

Through the use of a LED monitor, the mfERG stimulus can be kept constant during a stimulus of longer duration. Thus, the recording of on- and offresponses under stable luminance conditions becomes possible.

Therefore, we performed a photopic on-off mfERG using a LED stimulation screen in patients with normal-tension (NTG) and high-tension (HTG) POAG, as well as in a control group, in order to evaluate whether this stimulus is sufficiently sensitive to detect early glaucomatous dysfunction. 


\section{Methods}

Subjects

Fifteen NTG, fifteen HTG patients, and fourteen control subjects were included in this study. Procedures followed the tenets of the Declaration of Helsinki. The research protocol was approved by the Institutional Ethics Committee. Participants provided a written consent prior to the commencement of the examination.

All subjects underwent an ophthalmologic examination including visual acuity and measurement of intraocular pressure, slit-lamp biomicroscopy, and ophthalmoscopy.

Inclusion criteria for controls and glaucoma patients were the following:

- a best corrected Snellen visual acuity at distance better than 0.7 ,

- a refractive error within $\pm 6 \mathrm{dpt}$,

- an absence of previous ocular surgery,

- no history of systemic cardiovascular diseases, such as systemic hypertension or diabetes mellitus,

- no history of neurological diseases, and

- no ocular pathology other than glaucoma that may affect the mfERG recording.

Additional inclusion criteria for glaucoma patients were the following:

- a glaucomatous optic neuropathy, defined by an asymmetry in excavation, localized thinning of the neuroretinal rim of the optic disk, a cup-todisk ratio of at least 0.5 ,

- a reproducible glaucomatous visual field loss (MD worse than $-2.2 \mathrm{~dB}$, Octopus 101, G2 program), defined by a cluster of three points reduced by $5 \mathrm{~dB}$ or greater, and including at least one point reduced by $10 \mathrm{~dB}$ or greater; a cluster of two points reduced by $10 \mathrm{~dB}$ or greater; or three adjacent points on the nasal horizontal meridian that were reduced by $5 \mathrm{~dB}$ or greater from their mirror points on the opposite meridian.

All glaucoma patients had at least one diurnal tension curve without treatment (Goldman applanation tonometry at $6 \mathrm{am}$, before arising from bed, $8 \mathrm{am}, 11 \mathrm{am}, 4 \mathrm{pm}, 10 \mathrm{pm}$ ). Normal-tension glaucoma patients were defined as POAG patients having a highest ever measured intraocular pressure below $21 \mathrm{mmHg}$.

The left eye of each subject was selected for the study. If a subject's left eye did not qualify, the right eye was examined if it satisfied the inclusion criteria and none of the exclusion criteria (5 patients).

\section{On-off mfERG recording}

Before the recording session patients were adapted to ambient room light for approximately $30 \mathrm{~min}$. Pupils were maximally dilated (8-9 $\mathrm{mm}$ in diameter) with tropicamide $0.5 \%$ and phenylephrine $1 \%$ eye drops. The cornea was anesthetized with proxymetacaine hydrochloride. A ground electrode was attached to the center of the forehead. Retinal activity was recorded via a bipolar gold lens contact electrode (Dyagnosys LLC, Lowell, MA, USA). The lens was wetted with a drop of synthetic carbomer (ThiloTears $\mathrm{SE}^{\mathrm{R}}$, Alcon Pharma $\mathrm{GmbH}$ ) and placed on the anesthetized cornea. The opposite eye was occluded to avoid blinking. A corrective lens was not applied. The viewing distance was $28 \mathrm{~cm}$.

Retinal responses to on-off mfERG white stimuli were recorded monoculary under photopic conditions. The on-off mf ERG stimulation was generated on a light-emitting diode (LED) stimulus screen (RETIscan $^{\mathrm{TM}}$, Roland Consult system, GmbH, Wiesbaden) using a stimulus base interval of 200-ms duration (m-sequence stimulus, 100-ms LED-on or LED-off followed by 100-ms LED-off).

The stimulus array consisted of 1,024 $(32 \times 32)$ broadband white LEDs on a white background that stimulated the central $56^{\circ}$ of the retina. Each LED had a peak wavelength of about $424 \mathrm{~nm}$ with a relative luminous intensity of $100 \%$ and a half width (at $50 \%$ relative luminous intensity) of $28 \mathrm{~nm}$. There was a second smaller peak at about $550 \mathrm{~nm}$ with a relative luminous intensity of $40 \%$ and a respective half width (at $20 \%$ relative luminous intensity) of $120 \mathrm{~nm}$.

The LEDs were grouped into 61 hexagons. Hexagons were scaled with a distortion factor of 4 , in order to take into account the cone distribution within the central retina [30]. Thus, the central hexagon consisted of 4 LEDs and was smaller, whereas the most peripheral hexagons consisted of 16 LEDs and thus were four times larger. 
Hexagons flickered according to a pseudorandom sequence (frame rate: $70 \mathrm{~Hz}$ ). During the light phase (L) that lasted $100 \mathrm{~ms}$, the luminance of the hexagons was $180 \mathrm{~cd} / \mathrm{m}^{2}$. During the dark phase (D), the luminance of the hexagons was $0 \mathrm{~cd} / \mathrm{m}^{2}$, resulting in a contrast of $100 \%$. Mean luminance was $45 \mathrm{~cd} / \mathrm{m}^{2}$. The total recording time was $11.68 \mathrm{~min}$, subdivided into 8 segments of $1.46 \mathrm{~min}$ each. Retinal signals were bandpass filtered at $10-200 \mathrm{~Hz}$, and a spatial iteration was applied twice. The artifact rejection was preprogrammed on the RETIscan ${ }^{\mathrm{TM}}$ software.

In addition, all POAG patients underwent an Octopus (G2-program) visual field examination (Octopus model 101, Haag-Streit AG, Switzerland), where the central $60^{\circ}$ of the retina $\left(30^{\circ}\right.$ radius) was evaluated.

\section{Data analysis}

Figure 1 shows a representative overall response to long-duration white flashes of a control subject. There was a marked response initial negative and then positive (P1 or b-wave) to the stimulus onset (on-response) and a positive $\mathrm{P} 1$ or d-wave to the stimulus offset (off-response), each followed by a negative potential (N2-on, N2-off).

The N1, N1P1, and P1 responses would be expected to be generated primarily by the cone photoreceptors and the off- and on-bipolar cells (N1) $[26,27,31]$. In addition, in some of the recordings, the N1-off response was hardly visible. Thus, we

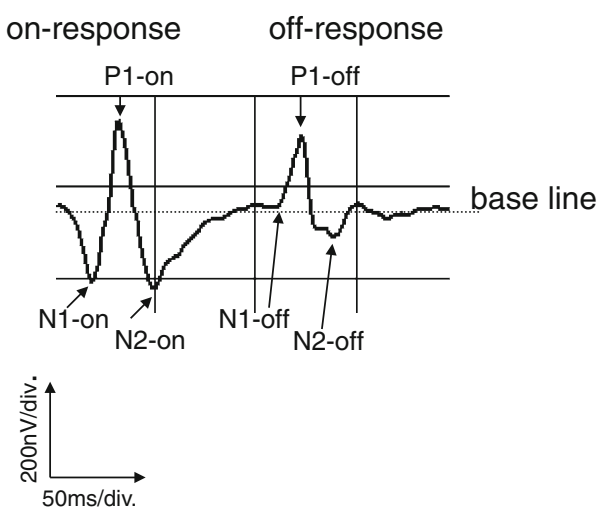

Fig. 1 A representative response of a control subject. There was a marked response to the stimulus onset (on-response) and to the stimulus offset (off-response). Both on- and offresponses showed a first negative (N1-on, N1-off), a first positive (P1-on, P1-off) peak, as well as a following second negative peak (N2-on, N2-off) concentrated analysis of our data on the second negative peaks, the $\mathrm{N} 2$ responses (N2-on, N2-off), which we assume also include information contained in the PhNR. Amplitudes of the N2 responses were measured from the baseline provided in the RETIscan $^{\mathrm{TM}}$ software to the second on- and off-negative peak. All measurements were taken manually.

For statistical analysis of the overall response, a oneway ANOVA was applied. If a $P$-value was $\leq 0.05$, this was followed by a post hoc test (Sidak). A repeated measure ANOVA allowing for correlation between locations (Huynh-Feldt) and adjusted for age was performed for the comparison of response averages. If the $P$-value was less than or equal to 0.05 , a post hoc (Sidak) test was performed. A Spearman bivariate test was used to correlate mfERG values with the mean defect values (MD) of the corresponding quadrants of the Octopus static perimetry, as well as with the mean variance of the local defect (LV).

\section{Results}

We recorded an on-off mfERG from 15 NTG, 15 HTG patients, and also from 14 control subjects.

Mean age, Snellen visual acuity, and IOP at the time of examination did not differ between the groups analyzed $(P>0.05$, multivariate ANOVA). The cup/ disk ratio differed slightly between NTG and HTG patients $(P=0.04)$, but both glaucoma groups differed significantly from the control group $(P=$ 0.00; one-way ANOVA, post hoc-Sidak).

Among all subjects, the IOP at the time of examination was below $21 \mathrm{mmHg}$. In 23 of 30 glaucoma patients, the IOP was controlled medically at the time of the examination.

Visual field parameters (mean MD, mean LV) did not differ between NTG and HTG patients. $(P>$ 0.10 , one-way ANOVA). Clinical details on the data of the glaucoma patients and the controls are presented in Table 1.

Analysis of the overall response

The overall response is the mean average response of the entire hexagon array (Fig. 1).

Figure 2 shows the box plots of the resulting overall N2-amplitudes and N2-latencies for the NTG, HTG, and the control group. 
Table 1 An overview of the clinical details of glaucoma patients and control subjects

\begin{tabular}{llllllll}
\hline Mean $(\mathrm{SD})$ & Age (years) & Eye tested & VA & IOP mmHg & C/D & MD dB & Gender \\
\hline Controls & $54.8(11.3)$ & $14 \mathrm{LE}$ & $1.0(0.1)$ & $13.4(2.6)$ & $0.3(0.0)$ & Not tested & 8:6 F:M \\
NTG & $54.9(7.2)$ & $1 \mathrm{RE} 14 \mathrm{LE}$ & $1.1(0.1)$ & $13.5(2.1)$ & $0.7(0.1)$ & $6.5(5.2)$ & $8: 7 \mathrm{~F}: \mathrm{M}$ \\
HTG & $62.3(9.5)$ & $4 \mathrm{RE} \mathrm{11} \mathrm{LE}$ & $1.0(0.1)$ & $14.8(3.3)$ & $0.6(0.1)$ & $4.0(2.3)$ & $5: 10 \mathrm{~F}: \mathrm{M}$ \\
Multivariate ANOVA $(P<0.05)$ & $P: 0.11$ & & $P: 0.43$ & $P: 0.33$ & $P: 0.00$ & $P: 0.10$ & \\
\hline
\end{tabular}

The NTG patients $(n=15)$, the HTG patients $(n=15)$, and the control subjects $(n=14)$ did not differ significantly in gender, age, visual acuity (VA), or intraocular pressure (IOP). Glaucoma patients did not differ significantly in mean MD

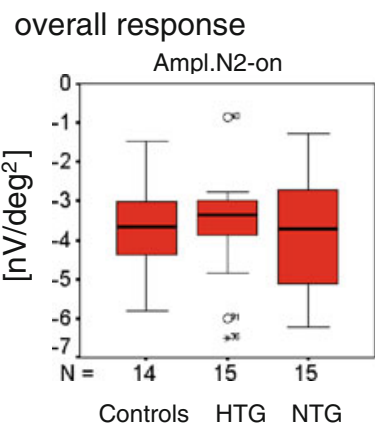

Fig. 2 The box plots of the resulting overall N2-amplitudes and latencies for the NTG, HTG, and the control group. The box length is the interquartile range. The median is presented
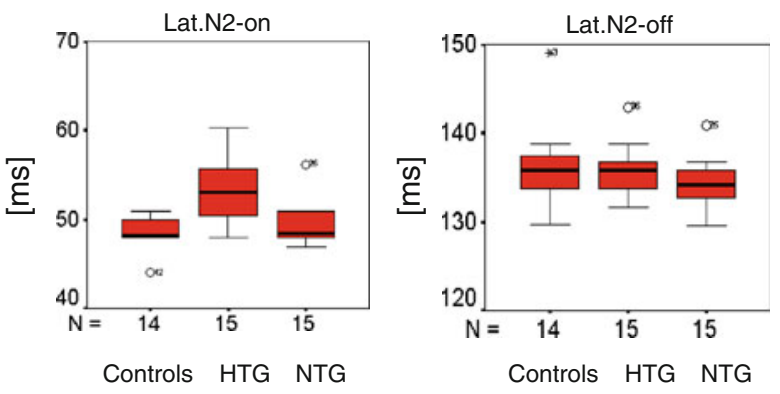

as a thick black line within the box. Cases with values between 1.3 and 3 box length are outliers (open circles) and those with values more than 3 box length are extremes (arrow heads)

Latencies of the N2-on differed significantly between groups $(P=0.001)$ in all quadrants examined, consistent with the results of the overall response. This was due to a delayed N2-on latency in HTG patients compared with controls $(P=0.001)$ and also with NTG patients $(P=0.048$, repeated measure ANOVA, post hoc-Sidak; Fig. 3, bottom). The NTG patients, however, did not differ significantly from the controls $(P=0.336)$. N2-off latencies did not differ significantly between the three groups $(P>0.05$, repeated measure ANOVA, post hoc-Sidak). There was no effect of location for the latencies of the N2-on $(P=0.70)$ and for the latencies of N2-off $(P=0.59$, ANOVA, HuynthFeldt, $P<0.05)$, nor did age have a significant influence $(P>0.05)$.

Amplitudes of $\mathrm{N} 2$ for glaucoma patients and controls did not show statistically significant differences for either the N2-on $(P=0.91)$ or the N2-off $(P=0.09$, repeated measure ANOVA, post hocSidak). There was also no influence of location for the amplitudes of the N2-on $(P=0.413)$, or the N2off $(P=0.96$, Huynh-Feldt).
Figure 3 (top) shows the area of responses that were grouped together to form response averages in quadrants. 
Latencies of the N2-on, quadrants

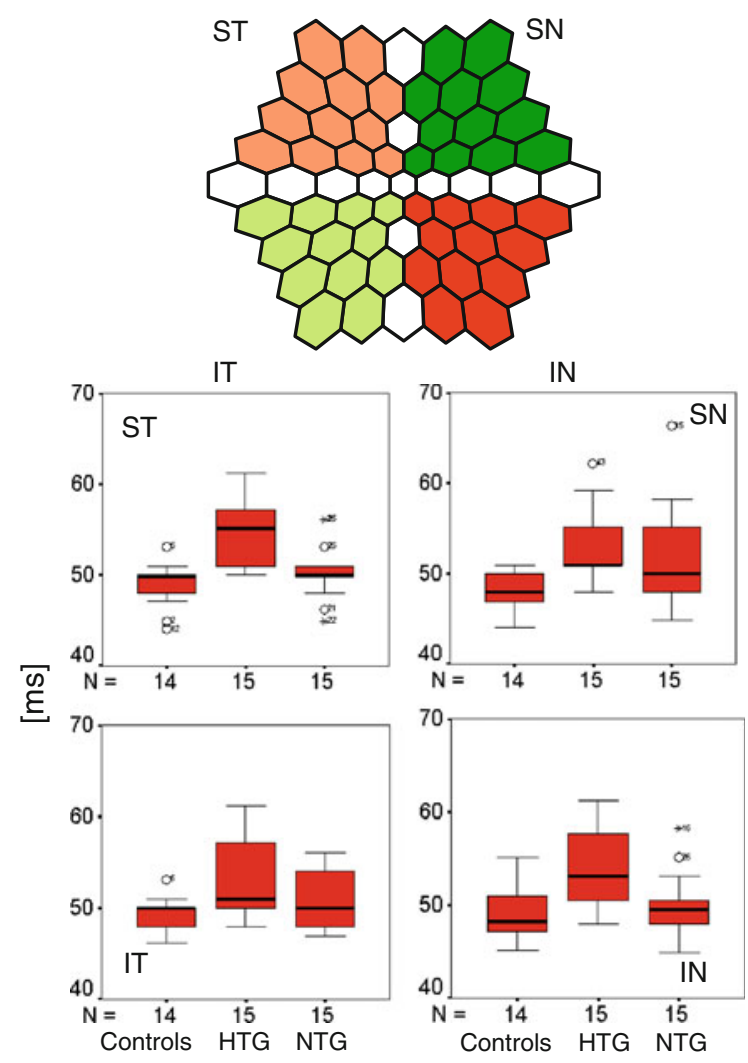

Fig. 3 Hexagons which were grouped together to form the response averages in quadrants, as well as box plots of the resulting N2-on latencies for the NTG, HTG and the control group are shown

Responses in 4 small peripheral locations

In order to test more peripheral locations focally, we averaged the local responses from 4 neighboring hexagons per quadrant from the more peripheral areas, without overlapping them.

Figure 4 shows the corresponding areas grouped together, as well as the box plots of the corresponding N2-on latencies, while Table 2 shows the corresponding mean latencies. When latencies of the N2on were analyzed, the HTG group differed significantly not only from the control group $(P=0.00)$ but also from the NTG group $(P=0.00$, repeated measure ANOVA, post hoc-Sidak). Again, there was no statistically significant difference between the NTG group and the control group $(P=0.99$, repeated measure ANOVA). Latencies of the N2-off did not differ between groups $(P>0.05$, repeated measure ANOVA).

Figure 5 shows the receiver operating characteristic (ROC) curve of the N2-on latencies of the small peripheral superior temporal quadrant response average for HTG patients. The area under the ROC of 0.88 (95\% CI: $0.79-1.00)$ indicates a high ability to differentiate between HTG and control $(P=0.00)$.

Again, there was no effect of location or influence of age for all parameters tested $(P>0.05$, ANOVA, Huynth-Feldt, $P<0.05$ ).

Responses in 4 small central locations

Retinal response averages were also examined for 4 small central locations in each quadrant (Fig. 6, top). Again, for the N2-on latencies, a statistically significant difference was found between the glaucoma group and controls. This held true not only for the HTG group $(P=0.000$, repeated measure ANOVA, post hoc-Sidak) but also for the NTG group $(P=0.04$, repeated measure ANOVA, post hoc-Sidak; Fig. 6, Table 2). NTG patients differed from the control group mostly in the small central response average of the inferior temporal quadrant. Here, the corresponding area under the ROC of 0.79 (95\% CI: 0.63-0.96) indicates an ability to differentiate between NTG and control, which is significantly higher than chance $(P=0.01)$.

The NTG patients, however, did not differ significantly from the HTG patients $(P=0.19)$.

There was no significant influence of age $(P>0.7)$ or location for the amplitudes or latencies of the N2-on and of N2-off $(P>0.05$, ANOVA, Huynh-Felt).

Relationship between the mfERG responses and the visual field defect

The diffuse retinal sensitivity loss, described by the mean defect (MD) is a logarithmic value adjusted for age. In order to evaluate the relationship between the visual field loss (MD) and the mfERG parameters, we converted the mfERG values to logarithmic units adjusted to the mean age (57.38). A Spearman bivariate test $(P<0.05)$ was used to correlate the mfERG parameters averaged in quadrants to the MD of the corresponding quadrant of the Octopus perimetry. 

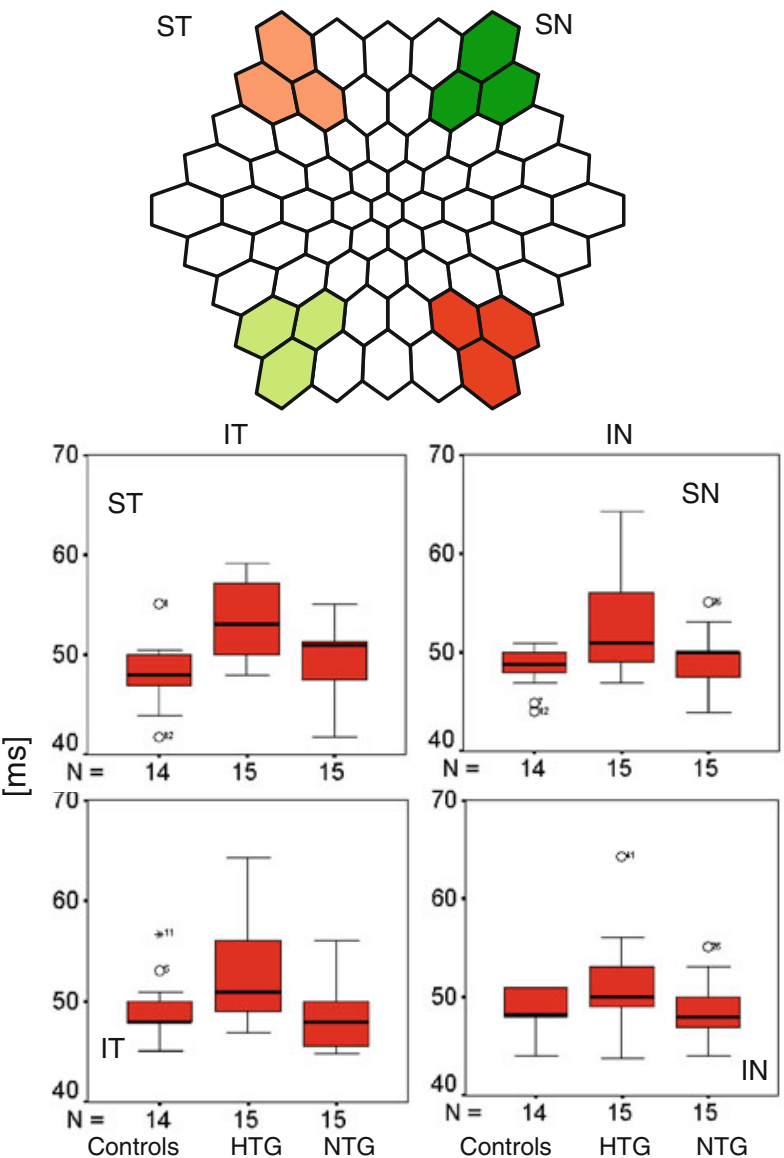

Fig. 4 Hexagons which were grouped together to form the small peripheral response averages in the respective quadrants (left top). To the right, representative responses from the small peripheral response averages in quadrants are presented for a

For both stimulus onset and stimulus offset responses, amplitudes of the N2 decreased, while the respective latencies increased as the MD got worse. However, these correlations did not reach a significance level (Spearman, Bonferroni).

Analyzing LV as an indicator for a local damage, NTG patients (mean $43.23 \mathrm{~dB}^{2}$ (SD 13.80)) seemed to be more affected than HTG patients (mean $18.51 \mathrm{~dB}^{2}$ (SD 20.14)). Both groups, however, did not differ significantly $(P=0.11$, one-way ANOVA). Also, a Spearman bivariate test showed no statistically significant correlation between the mfERG parameters and the LV of the Octopus perimetry $(P>0.05)$.
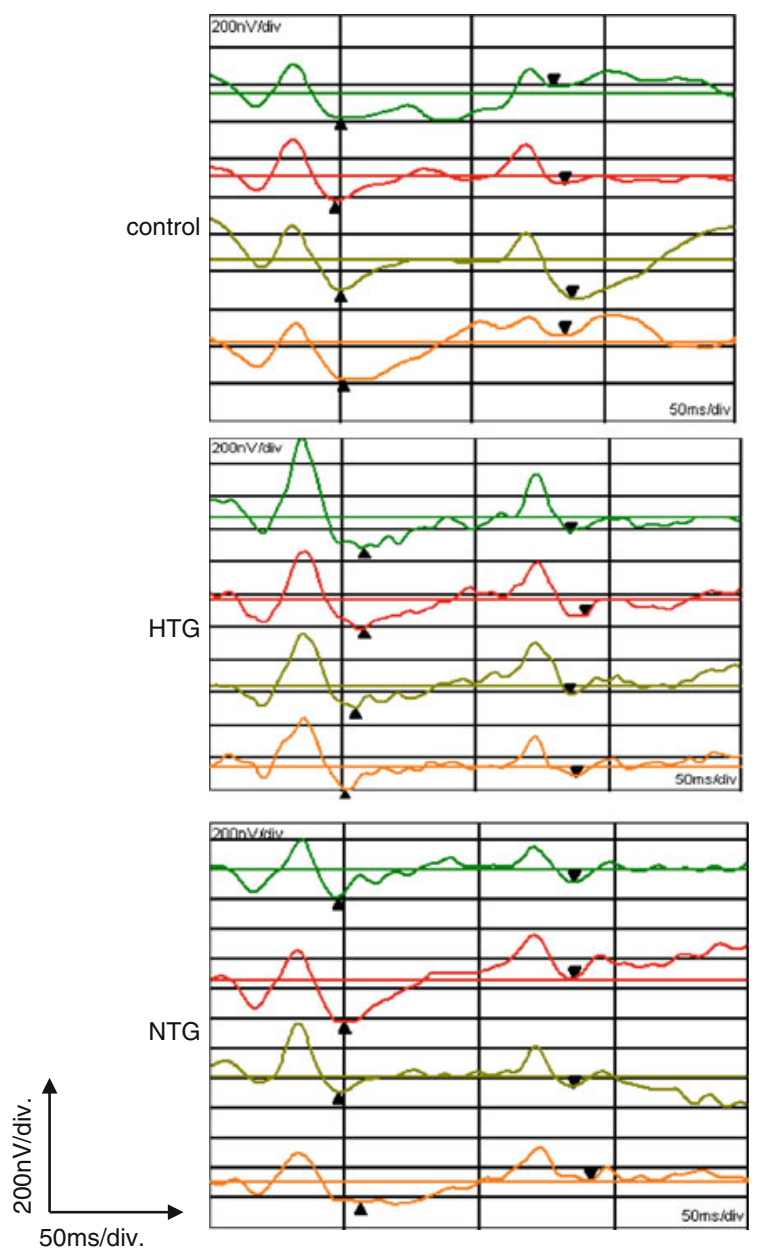

control subject, NTG, and HTG. Box plots (bottom left) depict the resulting N2-on latencies for the NTG, HTG, and the control group. The corresponding mean values and SD are given in Table 2 are shown

\section{Discussion}

In glaucoma, responses generated in the inner retina, especially the ganglion cells, would be expected to be primarily affected. As there is good evidence that the PhNR to both stimulus on- and the stimulus offset contains such inner retinal information and is affected in glaucoma [3, 4] we evaluated the negative troughs following the on- and off-responses to white photopic long-duration stimuli, the N2-on and the N2-off responses, respectively.

In the Ganzfeld ERG as well as in the uniform ERG, the implicit time of the PhNR has been suggested to be at around $100 \mathrm{~ms}$ in the on-response 
Table 2 The mean N2-on latencies and their SD for the responses averaged in 4 small peripheral and in 4 small central locations
Values in bold represent which latencies differed significantly from controls

\begin{tabular}{llll}
\hline Quadrants & Groups & $\begin{array}{l}\text { Small peripheral locations } \\
\text { Latencies N2-on [ms] } \\
\text { Mean(SD) }\end{array}$ & $\begin{array}{l}\text { Small central locations } \\
\text { Latencies N2-on [ms] } \\
\text { Mean(SD) }\end{array}$ \\
\hline Superior nasal & Controls & $48.6(2.2)$ & $48.4(2.5)$ \\
& NTG & $49.3(2.7)$ & $\mathbf{5 2 . 1}(\mathbf{4 . 0})$ \\
Inferior nasal & HTG & $\mathbf{5 3 . 5}(\mathbf{5 . 6})$ & $\mathbf{5 5 . 6}(\mathbf{7 . 2})$ \\
& Controls & $48.8(2.0)$ & $49.6(2.1)$ \\
Inferior temporal & NTG & $48.4(3.0)$ & $\mathbf{5 1 . 5}(\mathbf{4 . 4})$ \\
& HTG & $\mathbf{5 1 . 3}(\mathbf{4 . 8})$ & $\mathbf{5 4 . 6}(\mathbf{4 . 5})$ \\
& Nontrols & $49.0(3.1)$ & $49.5(3.1)$ \\
NTG & $48.5(3.3)$ & $\mathbf{5 3 . 6}(\mathbf{4 . 1})$ \\
& HTG & $\mathbf{5 2 . 7}(\mathbf{4 . 9})$ & $\mathbf{5 5 . 4}(\mathbf{6 . 4})$ \\
& Controls & $48.1(3.1)$ & $49.2(2.6)$ \\
& NTG & $49.4(3.6)$ & $\mathbf{5 3 . 3}(6.6)$ \\
& HTG & $\mathbf{5 3 . 6 ( 3 . 9 )}$ & $\mathbf{5 5 . 9}(6.1)$ \\
\hline
\end{tabular}

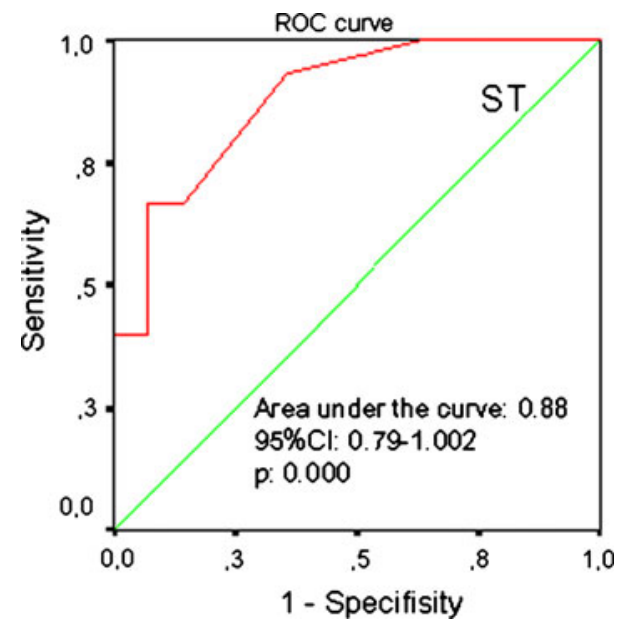

Fig. 5 The ROC curve based on the N2-on latencies of HTG patients for the small peripheral area in the superior temporal quadrant

and longer than $110 \mathrm{~ms}$ in the off-response [6]. In our study, the slow negative potential of the on-response, $\mathrm{N} 2$-on, developed at $48.7 \pm 1.8 \mathrm{~ms}$ and of the offresponse, N2-off, at $136.1 \pm 4.4 \mathrm{~ms}$ in controls. Although the negative troughs follow the on- and off-stimuli, similar to the PhNR, the latency was shorter under our stimulus and filter conditions. Nonetheless, we presume that these negative troughs may include information contained in the PhNR.

The findings of the present study show the latency of the N2-on to be significantly delayed in the HTG

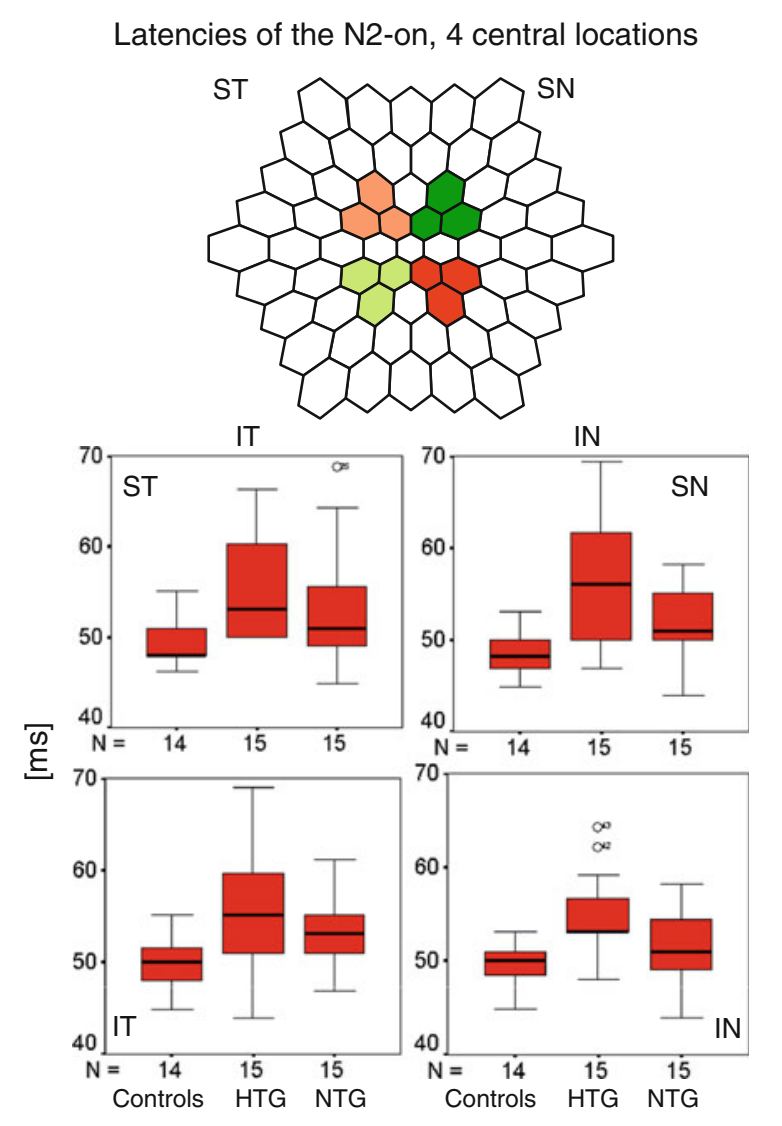

Fig. 6 Hexagons which were grouped together to form the small central response averages in the respective quadrants (left top). Box plots (bottom left) depict the resulting N2-on latencies for the NTG, HTG, and the control group are shown 
group when compared with controls. For NTG, this was also the case, but only in the overall response and in the small central response averages. As the latency of the PhNR has been found to increase by about $1.6 \mathrm{~ms}$ per decade [3], we took age into account when analyzing our N2-response data. Our findings were, however, not influenced by age.

In POAG, electrophysiological studies using the PERG have shown the frequent combination of localized, as well as of diffuse retinal damage in patients with glaucoma [32, 33]. For the full-field S-cone ERG, the PhNR has been reported to be able to differentiate patients with HTG but not with NTG from a control group [34]. For the mfERG with special stimulation parameters, small differences in the distribution and the amount of retinal dysfunction have been seen when HTG patients were compared with NTG patients [21, 35, 36].

In the present study, HTG patients also differed from NTG patients. The mfERG of the HTG patient group showed more diffuse retinal dysfunction, while the mfERG of the NTG patients group showed a more localized central retinal dysfunction. In the present study, HTG patients differed also morphologically from the NTG group in regard to the asymmetry in the excavation of the optic disk and to the LV as an indicator for a local damage, showing the NTG group to have more localized damage affecting predominantly the central zone compared with more diffuse and more peripheral damage in HTG patients. This is in agreement with the previous psychophysical studies, where when NTG patients were compared with HTG patients with a similar overall visual field loss, NTG patients showed more localized field defects, closer to the center of fixation [36].

In contrast to previous studies that reported a marked reduction in the PhNR amplitudes of the Ganzfeld ERG in experimental glaucoma and in glaucoma patients [3, 4, 8-10, 32, 37] and a marked reduction in amplitude with only a slight increase in the PhNR latencies using a slow stimulation mfERG paradigm [12], the present study showed no significant reduction in the amplitudes of the $\mathrm{N} 2$ responses in the white photopic long-duration on-off mfERG, but did find significant differences in latencies. These differences may be secondary to the stimulus parameters used, which differed between the studies in stimulus length, base interval, luminance, and the filter setting.
For instance, the shorter luminance step in our study compared with that used in Ganzfeld or unifield ERG [6] may not be enough for the PhNR to reach the full development, thus leading to prolongation of the N2-on implicit time in glaucoma patients without influencing the amplitude.

Also, in the present study, retinal responses to onoff mfERG stimuli were recorded using broadband white LEDs on a white background. In glaucoma, as well as in controls, a latency-delay of the PhNR-on to long-duration white-on-white stimuli has been found to be more significant [11] and the amplitude plateau less deep than to red stimuli on a blue background [9]. This could result in the PhNR-on-amplitude overlapping with the activity of the second-order hyperpolarizing retinal neurons and thus reduce its sensitivity to distinguish glaucoma patients from normals. For long-duration stimuli, the PhNR seemed to be better isolated using red flashes on a blue background, selectively stimulating the long-wavelength cones, but on the other hand, saturating the rods $[2,3,9,10]$.

Furthermore, in comparison with previous studies, analysis of the data was performed using the software included in RETIscan ${ }^{\mathrm{TM}}$. Here, the baseline goes through the mean of the entire response, which has an influence on the measurement of amplitudes.

A problem with the approach used lies in the filter setting applied. The RETIscan ${ }^{\mathrm{TM}}$ software is set at the filter setting recommended in the ISCEV guidelines [38]. This as a consequence means that responses with dominant frequencies below $10 \mathrm{~Hz}$ are removed. As the PhNR is a slow wave, the ideal low cutoff filter has been proposed to be around $0.3 \mathrm{~Hz}$ (maximum: $5 \mathrm{~Hz}$ ) $[4,5,14]$ in order to include the entire PhNR. For instance, with such a lower cutoff setting, the PhNR of the focal ERGs, in basic research and in clinical studies showed a marked reduction in amplitude in glaucoma $[4,5,13,14]$ even for the responses obtained from the central and paracentral retinal areas [14]. So, although the low-pass cutoff of $3-10 \mathrm{~Hz}$ is recommended in the ISCEV guidelines protocol mfERG [38], this filter seems to be inappropriate to isolate the $\mathrm{PhNR}$ in $\mathrm{mfERG}$ recordings with a slower stimulation. Thus, much of the PhNR which peaks at much later times in the long flash paradigm seems to be lost [13].

In conclusion, our results suggest that the N2-on response of the white photopic long-duration mfERG, 
in approximation to the $\mathrm{PhNR}$, is a sensitive feature that is affected in glaucoma. We did not find significant differences in the amplitude, but a significant latency delay of the N2-on response in POAG patients. In our patient group and under the stimulus parameters and filter setting applied, the latency delay of the N2-on was more sensitive for HTG than for NTG. For HTG, the most sensitive measure was the latency of the N2-on of the small peripheral response average of the superior temporal quadrant. For NTG, the most sensitive measure was the latency of the N2on of the small central response average of the inferior nasal quadrant.

The need for further studies on optimal stimulus paradigms, as well as on low-frequency cutoff filter settings, is acknowledged in order to find the optimal range for recording the PhNR in the clinical practice.

Acknowledgments We are grateful to Andy Schötzau for his advice on statistical analysis of the data. This study was supported by a grant from Pfizer (AP, MGT) and by a Swiss government grant for foreign students in Switzerland (MGT). The data were presented in part at ARVO 2005, EVER 2006, ARVO 2008.

\section{References}

1. Kuehn MH, Fingert JH, Kwon YH (2005) Retinal ganglion cell death in glaucoma: mechanisms and neuroprotective strategies. Ophthalmol Clin North Am 18:383-395

2. Viswanathan S, Frishman L, Robson JG, Harwerth RS, Smith EL III (1999) The photopic negative response of the macaque electroretinogram: reduction by experimental glaucoma. Invest Ophthalmol Vis Sci 40:1124-1136

3. Viswanathan S, Frishman L, Robson JG, Walters JW (2001) The photopic negative response of the flash electroretinogram in primary open angle glaucoma. Invest Ophthalmol Vis Sci 42:514-522

4. Colotto A, Falsini B, Salgarello T, Iarossi G, Galan ME, Scullica L (2000) Photopic negative response of the human ERG: losses associated with glaucomatous damage. Invest Ophthalmol Vis Sci 41:2205-2211

5. Machida S, Gotob Y, Toba Y, Ohtaki A, Kaneko M, Kurosaka D (2008) Correlation between photopic negative response and retinal nerve fiber layer thickness and optic disc topography in glaucomatous eyes. Invest Ophthalmol Vis Sci 49:2201-2207

6. Viswanathan S, Frishman LJ, Robson JG (2000) The uniform field and pattern ERG in macaques with experimental glaucoma: removal of spiking activity. Invest Ophthalmol Vis Sci 41:2797-2810

7. Weber AJ, Kaufmann P, Hubbard WC (1998) Morphology of single ganglion cells in the glaucomatous primate retina. Invest Ophthalmol Vis Sci 39:2304-2320
8. Morgan JE, Uchida H, Caprioli J (2000) Retinal ganglion cell death in experimental glaucoma. $\mathrm{Br} \mathrm{J}$ Ophthalmol 84:303-310

9. Rangaswamy NV, Shirato S, Kaneko M, Digby BI, Robson JG, Frishman LJ (2007) Effects of Spectral Characteristics of Ganzfeld Stimuli on the Photopic Negative Response $(\mathrm{PhNR})$ of the ERG. Invest Ophthalmol Vis Sci 48: 4818-4828

10. Sustar M, Cvenkel B, Brecelj J (2009) The effect of broadband and monochromatic stimuli on the photopic negative response of the electroretinogram in normal subjects and in open-angle glaucoma patients. Doc Ophthalmol 118:167-177

11. Cursiefen C, Korth M, Horn FK (2001) The negative response of the flash electroretinogram in glaucoma. Doc Ophthalmol 103:1-12

12. Fortune B, Wang L, Bui BV, Cull G, Dong J, Cioffi GA (2003) Local ganglion cell contributions to the macaque electroretinogram revealed by experimental nerve fiber layer bundle defect. Invest Ophthalmol Vis Sci 44:4567-4579

13. Kondo M, Kurimoto Y, Sakai T, Koyasu T, Miyata K, Ueno S, Terasaki H (2008) Recording focal macular photopic negative response (PhNR) from monkeys. Invest Ophthalmol Vis Sci 49:3544-3550

14. Machida S, Toba Y, Ohtaki A, Gotoh Y, Kaneko M, Kurosaka D (2008) Photopic negative response of focal electoretinograms in glaucomatous eyes. Invest Ophthalmol Vis Sci 49(12):5636-5644

15. Hood DC, Seiple W, Holopigian K, Greenstein V (1997) A comparison of the components of the multifocal and fullfield ERGs. Vis Neurosci 14:533-544

16. Sutter EE, Tran D (1992) The field topography of ERG components in man-I. The photopic luminance response. Vis Res 32:433-446

17. Bearse MA Jr, Sutter EE (1996) Imaging localized retinal dysfunction with the multifocal electroretinogram. J Optical Soc America 13:634-640

18. Bearse MA Jr, Sutter EE, Palmowski AM (1997) New developments toward a clinical test of retinal ganglion cell function. Vision Science and its Applications, vol 1. Optical Society of America, Washington DC, pp 280-283

19. Palmowski AM (2003) Multifocal stimulation techniques in ophthalmology-Current knowledge and perspectives. Strabismus 2:229-237

20. Palmowski AM, Ruprecht KW (2004) Follow up in open angle glaucoma. A comparison of static perimetry and the fast stimulation mfERG. Multifocal ERG follow up in open angle glaucoma. Doc Ophthalmol 108:55-60

21. Palmowski AM, Allgayer R, Heinemann-Vernaleken B (2000) The multifocal ERG in open angle glaucoma-a comparison of high and low contrast recordings in highand low-tension open angle glaucoma. Doc Ophthalmol 101:35-49

22. Hood DC, Bearse MA Jr, Sutter EE, Viswanathan S, Frishman LJ (2001) The optic nerve head component of the monkey's (Macaca mulatta) multifocal electroretinogram (mERG). Vision Res 41:2029-2041

23. Hood DC, Greenstein VC, Holopigian K, Bauer R, Firoz B, Liebmann JM, Odel JG, Ritch R (2000) An attempt to detect glaucomatous damage to the inner retina with the multifocal ERG. Invest Ophthalmol Vis Sci 41:1570-1579 
24. Chu PH, Chan HHL, Brown B (2006) Glaucoma detection is facilitated by luminance modulation of the global flash multifocal electroretinogram. Invest Ophthalmol Vis Sci 47:929-937

25. Shimada Y, Bearse MA Jr, Sutter EE (2005) Multifocal electroretinograms combined with periodic flashes: direct responses and induced components. Graefes Arch Clin Exp Ophthalmol 243:132-141

26. Sieving PA (1993) Photopic ON- and OFF-pathway abnormalities in retinal dystrophies. Trans Am Ophthalmol Soc 91:701-773

27. Sieving PA, Murayama K, Naarendorp F (1994) Push-pull model of the primate photopic electroretinogram: a role for hyperpolarizing neurons in shaping the b-wave. Vis Neurosci 11:519-532

28. Kondo M, Miyake Y, Horiguchi M, Suzuki S, Tanikawa A (1998) Recording multifocal electroretinogram on and off responses in humans. Invest Ophthalmol Vis Sci 39: 574-580

29. Kondo M, Miyake Y (2000) Assessment of local cone onand off-pathway function using multifocal ERG technique. Doc Ophthalmol 100:139-154

30. Curcio CA, Sloan KR, Kalina RE, Hendrickson AE (1990) Human photoreceptor topography. J Comp Neurol 292: 497-523

31. Hood DC, Frishman LJ, Saszik S, Viswanathan S (2002) Retinal origins of the primate multifocal ERG: implications for the human response. Invest Ophthalmol Vis Sci 43:1673-1685
32. Bach M, Pfeiffer N, Birkner-Binder D (1992) Pattern Electroretinogram reflects diffuse retinal damage in early glaucoma. Clin Vision Sci 7:335-340

33. Neppert B, Breidenbach K, Dannheim F, Hellner K (1996) Chronic open angle glaucoma: correlation of pattern electroretinography and visual field indices. Ophthalmologe 93:539-543

34. Aldebasi YH, Drasdo N, Morgan JE, North RV (2004) S-cone, L + M-cone, and pattern, electroretinograms in ocular hypertension and glaucoma. Vision Res 44:2749-2756

35. Palmowski-Wolfe AM, Allgayer R, Vernaleken B, Ruprecht KW (2006) Slow-stimulated multifocal ERG in high and normal tension glaucoma. Doc Ophthalmol 112: 157-168

36. Araie M (1995) Pattern of visual field defects in normaltension and high-tension glaucoma. Curr Opin Ophthalmol 6:36-45

37. Miyata K, Ueno S, Kondo M, Koyasu T, Terasaki H (2008) Comparison of photopic negative responses elicited by red and white xenon flashes in monkeys. Jpn J Ophthalmol 52:327-330

38. Hood DC, Bach M, Brigell M, Keating D, Kondo M, Lyons JS, Palmowski-Wolfe AM (2008) ISCEV guidelines for clinical multifocal electroretinography (2007 edition). Doc Ophthalmol 116:1-11 